\title{
Periodic Separating Reactors: Experiments and Theory
}

The novel combination of a pressure swing adsorber (PSA) with a periodic flow-forced packed-bed reactor is explored. The device provides integral component separation and reaction. Feed sequences studied for the periodic separating reactor (PSR) were those of rapid, single-bed pressure swing adsorption (RPSA). The experimental investigation employed $\mathrm{CO}$ oxidation over a packed bed of supported platinum catalyst and molecular sieve adsorbent. A reaction rate limited model is formulated and solved for a variety of irreversible and reversible reactions.

The presence of irreversible chemical reaction is shown to greatly enhance the separation achievable by RPSA alone. For a wide range of inlet $\mathrm{CO} / \mathrm{O}_{2}$ ratios, $\mathrm{CO}_{2}$ production could be increased up to two times over steady-state plug-flow reactor operation, while providing a recycle stream without phase change or extractive procedures. Selectivity and conversion improvements were predicted for multiple reaction systems. Other unusual features of operation, such as separation reversals, were also predicted and observed.

\author{
Garo G. Vaporciyan \\ Robert H. Kadlec \\ Department of Chemical Engineering \\ University of Michigan \\ Ann Arbor, Ml 48109
}

\section{Introduction}

Numerous studies have shown, both experimentally and theoretically, that benefits can arise from imposing a repetitive discontinuity on an otherwise continuous operation. Reviews exist on the subject of periodic reactor operation (Bailey, 1977) and cyclic separation processes (Wankat, 1974; Schrodt, 1967). Significant throughput increases, lower energy input, economical small-scale operation, selectivity alterations, and increased conversions are among the improvements most often noted. Recent studies concerning periodic operation of process units have centered around concentration-forced reactors and pressure swing adsorption. Only a few cyclic separating reactor schemes have been investigated: pulsed chromatographic reactors and reactive parametric pumping.

In this paper the cyclic separation process of pressure swing adsorption (PSA) is combined with periodic reactor operation. The process cycle is similar to PSA, but feed components are simultaneously reacted and separated. Although such a hybrid device could utilize any of the multistep multibed, single-bed, or rotational forms of PSA, only the rapid-cycle single-bed version (RPSA) is considered here, Figure 1. The column contains a

\footnotetext{
The present address of G. G. Vaporciyan is Shell Development Company, Westhollow Research Center, Houston, TX 77251-1380.
}

mixture of catalyst (of the reaction requires such activation) and adsorbent. The gaseous reactant is pumped into the packed bed under high pressure for a fraction of the operating cycle. The feed end may be dead-ended before being allowed to exhaust during the rest of the process cycle. Large time-varying pressure waves exist near the feed end but, due to the low permeability of the packing, a continuous stream may be removed at the delivery end of the column. A surge volume is placed between the reaction bed and the delivery stream to allow for reflux (purge gas), and perhaps higher product purities as was found for RPSA (Turnock and Kadlec, 1971). After a startup period consisting of many cycles, a stationary (repetitive) cyclic steady state is reached, with periodically varying pressure and composition existing in the bed. The chemical conversion and separation accomplished depend upon the manner in which the pressure and reactant concentrations are controlled at the feed boundary.

Several types of pressure and reactant cycling, such as sinusoidal, triangular, and square-wave, are possible. Bang-bang pressure control was selected because:

1. Bang-bang feed pressure control of the underlying RPSA system was found to be optimal (Kowler and Kadlec, 1972)

2. In a number of other periodic processes the optimal control policy has been shown to be bang-bang (Horn and Lin, 1967) 

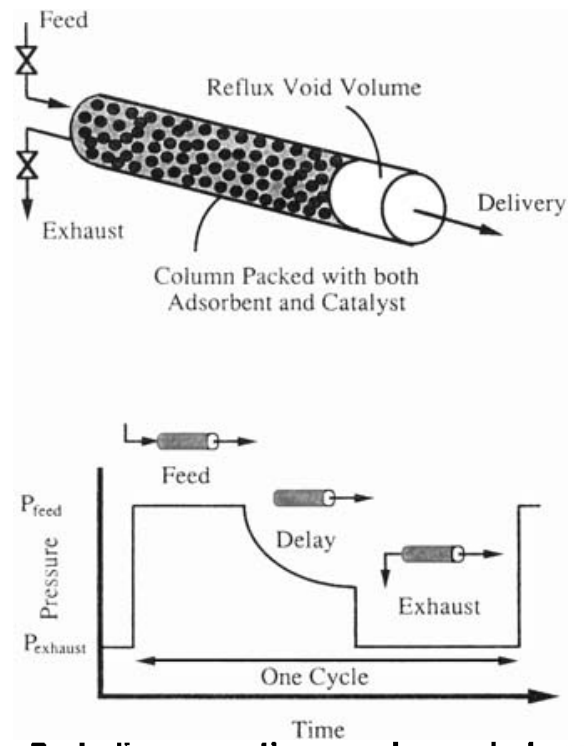

Figure 1. Periodic separating reactor and characteristic inlet pressure wave form.

3. Square waves have been found superior to sine waves for CO oxidation on alumina-supported platinum (Barshad, 1985)

4. Square-wave cycling was indicated as optimal for chromatographic reactors (Chu and Tsang, 1971)

5. On-off control is the easiest to implement

During the feed phase of a cycle, various concentrations patterns may be employed. Two bounds are: introduction by stages, one reactant after another, and introduction as a constant composition mixture. The first method would create a periodic separating reactor (PSR) in which both reactant concentration forcing and flow rate cycling are being imposed. Concentration cycling is not completely absent in the second scheme due to separation of the different reactants by the adsorbent.

The objective of this study was to obtain a better understanding of the mechanism and behavior of the periodic process for various reactions within a specific operating region: isothermal, reaction rate limited operation for constant feed compositions. Both experiments (with a specific catalyzed reaction) and modeling studies (for numerous irreversible and reversible reactions) were performed. Selection of the carbon monoxide oxidation reaction was dictated by the availability of analytical equipment, adsorbent, catalyst knowledge, and transient reactor operational experience (Vaporciyan et al., 1988) rather than the potential for performance improvements. Experiments permit model verification, while simulations allow expeditious investigation of a broad range of reactions for possible performance enhancements.

It is significant that this process has no steady-state analogue and therefore cannot be described against the background of steady-state theory. The performance of a reaction rate limited PSR can however, be described by RPSA, reaction equilibrium limited PSR, and chromatography effects. Experimental observations and results were compared with those predicted by a mathematical model that assumed instantaneous sorption equilibrium and reaction rate limited performance. Qualitative and quantitative agreement was noted, with all model parameters obtained via independent non-PSR experiments.

Many parameters that affect the separation and conversion performance of a PSR system were investigated. The capacity performance for reaction rate limited PSR is the same order of magnitude as equilibrium RPSA. Several different operating regions were observed in which separations greater than that possible with RPSA could be achieved. Phenomena such as separation reversals and the movement of a sharp reaction front were also observed. For a wide range of inlet $\mathrm{CO} / \mathrm{O}_{2}$ ratios carbon dioxide production could be enhanced up to two times over conventional steady-state plug-flow reactor operation. Selectivity and conversion improvements over conventional reactor operation were predicted for multiple reaction systems. A PSR may provide a recycle stream without phase change or extractive procedures while reducing catalytic requirements for production units. In short, new operating regions are possible, which are not accessible with RPSA or a steady state catalytic reactor.

\section{Experimental Details}

The experimental results for the PSR relate to a $157.5 \mathrm{~cm}$ long, $2.1 \mathrm{~cm}$ ID plain carbon steel pipe packed with both platinum catalyst beads and $5 \AA$ molecular sieve. The effect of pressure swings on the pellet effectiveness factor for various reaction kinetics has been investigated (Hamer and Cormack, 1978) and found to be significant under certain conditions. To eliminate such effects, an eggshell catalyst was employed. The catalyst was $0.5 \mathrm{wt}$. \% platinum deposited on the surface of $0.348 \pm$ $0.033 \mathrm{~cm}$ Grace low-density $\Theta$ alumina spherical beads. Techniques and criteria for producing eggshell, among other distributions (egg white, egg yolk, uniform), are known (Lee and Aris, 1985). The nonaqueous impregnation technique and alumina support were provided by General Motors Corp. At high loading, such as $0.5 \mathrm{wt}$. \%, an aqueous procedure would spread the metal uniformly throughout the beads. Catalyst deactivation was not observed during the course of the experiments.

The PSR bed was prepared by first packing with the catalyst spheres $(229.6 \mathrm{~g})$ and then introducing the Davison $5 \AA$ molecular sieve ( $143.1 \mathrm{~g}$ ). Use of 32-48 mesh round adsorbent particles and vibration insured that the interstitial voids between the much larger catalyst beads would be completely filled and that attrition of the adsorbent particles would not be a problem as it was in early work (Turnock and Kadlec, 1971). The procedure was tested with a $2 \mathrm{~cm}$ ID glass pipe. Measurement of permeabilities over $30 \mathrm{~cm}$ intervals of the bed before each experimental series provided repeated checks of the packing. No attrition of the bed occurred over the course of the experiments. The bed was mounted at an incline of about 35 degrees, to eliminate the possibility of a void channel developing along the top of the bed. Zonal temperature control was achieved with external electrical heat sources. During the preparation of the adsorbent bed some moisture from the air inevitably contaminated the molecular sieve. Vacuum evacuation and purge gas stripping at $445 \mathrm{~K}$ were utilized to remove this adsorbed water.

The processing scheme is illustrated in Figure 2. Due to the exothermicity of the reaction, nitrogen was employed as an inert heat sink. The reactant feed mixture was produced by blending of three different sources: liquid nitrogen, $\mathrm{CP}$ grade $\mathrm{CO}$, and dry air. Nitrogen concentrations in the feed were never below $90 \mathrm{~mol}$ $\%$. Blending was accomplished at the regulated pressure. Feed rotameters allowed for repeatability, but concentration measurement was employed to characterize the feed mixture. Surge vessels were used for both the inlet and outlet flow. The feed 


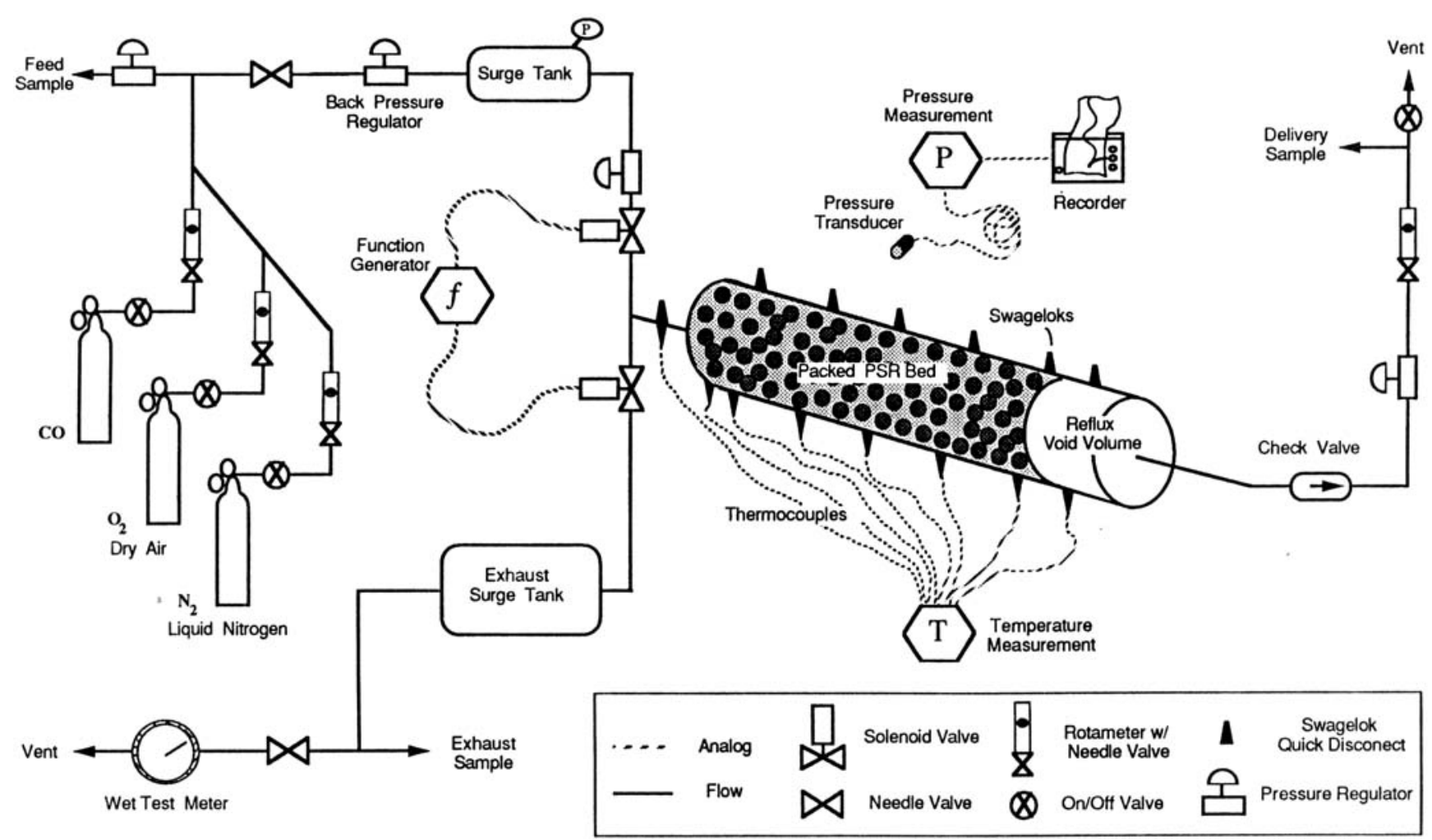

Figure 2. Experimental periodic separating reactor system.

mixture passed through a $820 \mathrm{~cm}^{3}$ surge vessel, and the exhaust flowed through a $3,600 \mathrm{~cm}^{3}$ surge vessel before flow measurement. The forward pressure regulator between the feed surge tank and the feed solenoid valve was set for the desired feed pressure. All other flow resistances were kept to a minimum. The solenoid valves were chosen with a $6.35 \mathrm{~mm}$ internal orifice, which presented very little resistance to flow. The only other obstacle to flow between the PSR feed pressure regulator and the packed bed was one thickness of 80 mesh screen used to support the adsorbent-catalyst blend.

The desired cyclic pressure wave form was produced with two high-temperature two-way solenoid valves, using the following cycling sequence:

1. Feed valve open, exhaust valve closed

2. Both valves closed

3. Feed valve closed, exhaust valve open

Valve wear was not a problem even after $2,500 \mathrm{~h}$ of almost continuous use at temperatures averaging over $420 \mathrm{~K}$. The valves were electrically controlled by a circuit consisting of two programmable timer relays, with a repeat accuracy of $\pm 0.1 \%$.

To achieve the desired feed boundary pressure control, care must be taken that the closing of the exhaust valve exactly coincides with the opening of the feed valve. If there is any time that both valves are simultaneously open, gas will bypass the column and flow directly from the feed to the exhaust. Despite the precautions taken to prevent such an event, bypass did occur and in some runs amounted to almost $10 \%$ of the total exhaust stream flow. The experimental data were corrected for this measured effect.

Exhaust gas pulses were damped by the surge vessel, and total flow measured with a wet test meter. The delivery line pressure regulator provided complete damping of the attenuated pressure pulsations of the delivery gas so that a rotameter was used to measure the flow rate. By calibration of the delivery rotameter against a bubble flow meter, the relative uncertainty of a delivery flow rate measurement was within $1.0 \%$. The delivery flow rate was regulated by a needle valve immediately preceding the rotameter. The total feed flow rate was maintained by setting the needle valve downstream of the feed rotameters such that the pressure swing in the feed surge tank remained constant.

The concentration measurement system consisted of an oxygen electrochemical cell monitor, a $\mathrm{CO}_{2}$ nondispersive infrared (NDIR) analyzer, and a CO NDIR analyzer in series. Introduction of calibration gases, samples, and dilution of the sample stream could all be accomplished easily. All flow rates and concentrations were measured, and both carbon and oxygen balances were computed. These typically resulted in a relative uncertainty of $\mathrm{CO}$ conversion of $3 \%$, and stream compositions of $2 \%$. Scatter in the experimental data points is representative of the inability to control set points of PSR parameters during an experimental series, rather than the inability to measure performance accurately.

Properly designed ports along the bed allowed access for both gas sampling and pressure response measurements. Quick response chromel-alumel thermocouple probes $(0.25 \mathrm{~mm})$ along the column provided temperature data.

The PSR wall temperature, feed composition, feed gas pressure, delivery flow rate, cycling frequency, and feed and delay fractions were all controlled during an experiment. The exhaust and delivery gas rates and compositions along with thermocouple response were all periodically measured during an experimental run. In some instances the compositions of gas samples and pressure histories along the bed were also measured. Cyclic 
steady state was assumed when atom balances closed within experimental error for at least $2 \mathrm{~h}$.

Further details may be found in the thesis of Vaporciyan (1987).

\section{Reaction Rate Limited Model}

Modeling of separating reaction systems usually takes the approach of considering the separation portion of the process at local equilibrium, while employing a macroscopic law for the determination of reaction rates. Modeling of periodically operated heterogeneous reactors has focused on rate imporvements based on the adsorption-desorption phenomena and nonlinear interactions on the catalyst surface. Models have been developed that include the presence of inactive or additional surface species, poisoning, cluster islands, surface diffusion, coveragedependent changes in the catalyst, or other nonlinearities and complications (Razon and Schmitz, 1986).

Meanwhile, the performance of one-bed rapid PSA (RPSA) has been successfully modeled (Turnock and Kadlec, 1971) and optimized (Kowler and Kadlec, 1972) for two component separations. Extension of their model to a multicomponent system has also been accomplished (Vaporciyan, 1987).

Within a periodic separating reactor, even when adsorption and desorption rate phenomena are considered at local equilibrium, heterogeneous surface concentrations are not constant with respect to time. Due to fluctuating pressures, modeling must include accumulation terms for both gas and solid phases, even at cyclic steady state. If local reaction rates are fast enough, local reaction equilibrium may be assumed. As with sorption equilibrium, local reaction equilibrium will vary cyclically. Such a perturbed equilibrium model was previously investigated (Vaporciyan and Kadlec, 1987).

For the pressure swing adsorption system employed by Turnock and Kadlec (which is the same type employed in the version of PSR developed here), extensive comparisons of computed and experimental results presented no evidence that the periodic process at $24^{\circ} \mathrm{C}$ is anything but an adsorption equilibrium process. It then seems reasonable to retain the assumption of sorption equilibrium and instead relax the reaction equilibrium constraint from the perturbed equilibrium model; thus a reaction rate limited PSR model.

Consider a periodic separating reactor in which $J$ reactions proceed involving $N$ components. Formulation of the mathematical model begins with these simplifying assumptions:

1. Local reaction rates determined from gaseous partial pressures

2. Local sorption equilibrium

3. Isothermal operation

4. No radial concentration and/or pressure gradients

5 . The process is not diffusion limited in any respect

6. Ideal gas behavior

7. Darcy's law for flow through packed beds

8. Linear sorption isotherm

9. Ideal solution behavior in the gas phase

10. Perfect pressure response during feed/exhaust and exhaust/feed switches

11. No variations in the feed concentration

12. Well-mixed void volume at the delivery end of the reactor
13. No void volume at the feed end of the packed bed

14. Constant molar delivery stream flow rate

The construction of the PSR was such that most of the above assumptions are correct. Assumptions 2-14 are valid for operation of a single-bed pressure swing adsorber without reaction at sufficiently high temperatures (Turnock and Kadlec, 1971). The reaction rate limited assumption would be valid for a wide range of temperatures and thus represents one operating region for PSR's. For the experimental PSR the exothermality of the $\mathrm{CO}$ oxidation reaction is compensated by the nitrogen diluent, and the external heat source. The diluent also helps to assure the correctness of assumption 8 , while the eggshell catalyst minimizes diffusion resistances. The volume between the feed/ exhaust solenoids and the packed bed was minimized by design.

A single reaction (of the $J$ present) is considered of the form $\Sigma v_{i} S_{i}=0$. The state equations may be formed from conservation laws and thermodynamics. After being made dimensionless, a mole balance for each component leads to the following set of equations:

$$
\left(\frac{\tau P_{f} k}{\mu L^{2}}\right) \frac{\partial}{\partial \lambda}\left\{Z_{i} \frac{\partial Z}{\partial \lambda}\right\}+\frac{r_{j} \rho_{C} R T_{\tau}}{P_{f}}=\gamma_{i} \frac{\partial Z_{i}}{\partial \theta} \quad \text { for } i=1,2, \ldots, N
$$

where the dimensionless capacity is:

$$
\gamma_{i}=\epsilon+\rho_{A} R T \alpha_{i} \text { for } i=1,2, \ldots, N
$$

and the total dimensionless pressure is determined from:

$$
Z=\sum_{i=1}^{N} Z_{i}
$$

The net reaction rate for each component is:

$$
r_{j i} v_{j i}=r_{j l} v_{j l} \text { for } j=1,2, \ldots, J, \quad i=1,2, \ldots, N
$$

where $l$ is the reference component for reaction $j$. Stochiometry yields:

$$
r_{i}=\sum_{j=1}^{J} r_{j i} \text { for } i=1,2, \ldots, N
$$

The rate law for each reaction would take the form,

$$
r_{j l}=f\left(Z_{i}^{\prime} s ; \bar{k}\right) \text { for } j=1,2, \ldots, J
$$

For the non-CO oxidation simulations rate laws are assumed to follow the elementary homogeneous reaction expression:

$$
r_{j l}=\mathscr{K}_{j} P_{f}^{\beta_{j}} \prod_{i=1}^{N} Z_{i}^{\beta_{j}}-\frac{\mathscr{K}_{j}}{K_{\text {eqj }}} P_{f}^{\sigma_{j}} \prod_{i=1}^{N} Z_{i}^{\sigma_{j i}} \quad \text { for } j=1,2, \ldots, J
$$

The form of the rate equation used in the $\mathrm{CO}$ oxidation model has been obtained from Hegedus et al. (1977):

$$
r_{\mathrm{CO}}=\frac{\left[\frac{a K_{1}}{T} \exp (-E / R T)\right] P_{\mathrm{Co}} P_{\mathrm{O}_{2}}}{\left\{1+\left[K_{2} \exp (H / R T)\right] P_{\mathrm{Co}}\right\}^{2}}
$$


where the local Pt surface area for the eggshell catalyst is determined from the equation presented by Oh et al. (1980):

$$
a=2.6 \times 10^{6}\left(\frac{\Delta^{3}}{\Delta^{3}-\delta^{3}}\right) D C
$$

Although this rate equation has not been tested under periodic conditions, it has the advantages of having measurable parameters, being based upon a Langmuir-Hinshelwood type expression, and having been successfully employed to predict stable isothermal multiplicities observed experimentally during carbon monoxide oxidation over a similar batch of alumina-supported platinum catalyst.

The above $(3 N+J N+J+1)$ state equations, Eqs. $1-7$, are the basis of the model, along with time and boundary conditions, and similar reflux void volume conservation balances. For the reflux void volume the following mole balances apply. When flow is from the packed bed into the reflux void volume, that is,

$$
\begin{aligned}
\text { when }\left.\frac{\partial Z}{\partial \lambda}\right|_{\lambda=1}<0 \quad \text { then for } i=1,2, \ldots, N \\
. \frac{\partial Z_{R i}}{\partial \theta}=-\left.\left(\frac{\tau P_{f} k}{\mu L^{2}}\right)\left(\frac{A L}{V_{R}}\right) Z_{i} \frac{\partial Z}{\partial \lambda}\right|_{\lambda=1}-\left(\frac{Q_{d} R T \tau}{P_{f} V_{R}}\right)
\end{aligned}
$$

During reflux, for flow from the void volume into the packed bed, that is,

$$
\begin{aligned}
\text { when }\left.\frac{\partial Z}{\partial \lambda}\right|_{\lambda=1} \geq 0 \quad \text { then for } i=1,2, \ldots, N \\
\cdot \frac{\partial Z_{R i}}{\partial \theta}=-\left.\left(\frac{\tau P_{f} k}{\mu L^{2}}\right)\left(\frac{A L}{V_{R}}\right) Z_{R i} \frac{\partial Z}{\partial \lambda}\right|_{\lambda-1}-\left(\frac{Q_{d} R T \tau}{P_{f} V_{R}}\right)
\end{aligned}
$$

A mole fraction constraint exists:

$$
Z_{R}=\sum_{i=1}^{N} Z_{R i}
$$

Boundary conditions for the packed bed are:

At the feed end, $\lambda=0$, during the feed portion of the cycle ( from $\theta=0$ to $\theta=f_{f}$ ),

$$
Z(0, \theta)=1, \quad Z_{i}(0, \theta)=Z_{f, i} \quad \text { for } i=1,2, \ldots, N
$$

during the delay portion of the cycle (from $\theta=f_{f}$ to $\theta=f_{f}+f_{D}$ ),

$$
\left.\frac{\partial Z}{\partial \lambda}\right|_{\lambda-0}=0
$$

and during the exhaust portion of the cycle (from $\theta=f_{f}+f_{D}$ to $\theta=1$ ),

$$
Z(0, \theta)=Z_{\min }
$$

At the delivery end, $\lambda=1$, during reflux into the packed bed from the void volume, that is,

$$
\begin{aligned}
& \text { when }\left.\frac{\partial Z}{\partial \lambda}\right|_{\lambda=1}>0 \text { then, } Z(1, \theta)=Z_{R}(\theta), \\
& Z_{i}(1, \theta)=Z_{R, i}(\theta) \text { for } i=1,2, \ldots, N
\end{aligned}
$$

Cyclic steady state is achieved when the conditions within the packed bed and reflux volume repeat every cycle. These periodicity conditions are:

$$
\begin{array}{ll}
Z(\lambda, \theta)=Z(\lambda, \theta+1) \quad \begin{array}{l}
Z \\
Z
\end{array}(\lambda, \theta)=Z_{i}(\lambda, \theta+1) \\
& \text { for } i=1,2, \ldots, N \\
Z(\lambda, \theta)=Z(\lambda, \theta+1) \quad Z_{R, i}(\lambda, \theta)= & Z_{R, i}(\lambda, \theta+1) \\
& \text { for } i=1,2, \ldots, N
\end{array}
$$

The total dimensional cycle average feed rate is calculated from:

$$
Q_{f}=\left.\left(\frac{A L P_{f}}{\tau R T}\right)\left(\frac{\tau P_{f} k}{\mu L^{2}}\right) \int_{0}^{f_{f}} \frac{\partial Z}{\partial \lambda}\right|_{\lambda=0} d \theta
$$

Individual component feed rates are determined from:

$$
\begin{aligned}
Q_{f, i}=\left.\left(\frac{A L P_{f}}{\tau R T}\right)\left(\frac{\tau P_{f} k}{\mu L^{2}}\right) Z_{f, i} \int_{0}^{f_{f}} \frac{\partial Z}{\partial \lambda}\right|_{\lambda-0} d \theta & \text { for } i=1,2, \ldots, N
\end{aligned}
$$

The total dimensional cycle average exhaust rate is calculated from:

$$
Q_{e}=\left.\left(\frac{A L P_{f}}{\tau R T}\right)\left(\frac{\tau P_{f} k}{\mu L^{2}}\right) Z_{\min } \int_{f_{f}+f_{0}}^{1} \frac{\partial Z}{\partial \lambda}\right|_{\lambda=0} d \theta
$$

The individual component exhaust rates are determined from:

$$
\begin{array}{r}
Q_{e, i}=\left.\left(\frac{A L P_{f}}{\tau R T}\right)\left(\frac{\tau P_{f} k}{\mu L^{2}}\right) \int_{f_{f}+f_{D}}^{1} Z_{i} \frac{\partial Z}{\partial \lambda}\right|_{\lambda=0} d \theta \\
\text { for } i=1,2, \ldots, N
\end{array}
$$

Although the delivery molar flow rate is constant during a cycle and equal to $Q_{d}$, individual component delivery flow rates are determined from:

$$
Q_{d, i}=Q_{d} \int_{0}^{1} Z_{R, i} d \theta \quad \text { for } i=1,2, \ldots, N
$$

This resulting reaction rate limited model contains $N$ secondorder, nonlinear, partial differential equations, along with $(2 N+J N+J+1)$ algebraic equations of which up to $J$ algebraic equations may be nonlinear. Independent variables are the dimensionless length down the reactor, $\lambda$, and dimensionless cycle time, $\theta$. Dependent variables are the dimensionless pressures $Z$ and $Z_{R}$, and partial pressures $Z_{i}$ and $Z_{R, i}$ in both the PSR and reflux void volume. The boundary conditions are time and state dependent, and include ordinary differential equations.

An algorithm for solving the reaction rate model for any arbi- 
trary reaction rate expression has been written and is described elsewhere (Vaporciyan, 1987). A cell discretization was employed. It was determined that exhaust and delivery stream flow rates are invariant to the number of cells, once above five. Up to eleven cells were employed for the results presented here. Predicted exhaust and delivery stream flow rates were found to be within $5 \%$ of experimental values. On an Amdahi 470 V/8 computer, execution times to reach cyclic steady state were on the order of $20 \mathrm{CPU}$ min. Execution times for $\mathrm{CO}$ oxidation stimulations were at least ten times longer than those employing elementary homogeneous rate laws. For the systems investigated, initial conditions did not affect the cyclic steady state solution achieved. In addition, perturbations from a cyclic steady state resulted in dynamics that returned the PSR to the original cyclic steady state.

\section{Dimensional analysis}

The dimensionless parameters that characterize the reaction rate limited PSR are shown in Table 1. The PSR Damköhler number is that for conventional reactors, with the process cycle period replacing the reactor residence time. The pressure dependence of the reaction equilibrium is contained in the dimensionless equilibrium constant. For the $\mathrm{CO}$ oxidation model the dimensionless equilibrium constant is zero, and the PSR Damköhler number is replaced by two dimensionless groups that arise as a result of the form of the oxidation rate law. The first is that employed for an irreversible second-order reaction, while the second is due to the inhibition term. The dimensionless

Table 1. Dimensionless Groups and Parameters for Reaction Rate Limited Periodic Separating Reactor Model

\begin{tabular}{|c|}
\hline Dimensionless Groups \\
\hline \multirow{2}{*}{$\begin{array}{l}\text { PSR Damköhler number } \\
\qquad\left[\epsilon_{f} P_{j}^{\left(\beta_{j}-1\right)} p_{c} R T \tau\right] \text { for } j=1,2, \ldots, J\end{array}$} \\
\hline \\
\hline $\begin{array}{l}\text { Dimensionless equilibrium constant } \\
\qquad\left[\frac{P_{f}^{\left(\sigma_{j}-\beta_{j}\right)}}{K_{\text {eq, } j}}\right] \text { for } j=1,2, \ldots, J\end{array}$ \\
\hline Dimensionless capacity \\
\hline$\left(\epsilon+\rho_{A} R T \alpha_{i}\right)$ \\
\hline Pressure ratio \\
\hline $\begin{array}{l}\text { Cycle time/reflux residence time ratio } \\
\qquad\left(\frac{Q_{d} R T_{\tau}}{P_{f} V_{R}}\right)\end{array}$ \\
\hline $\begin{array}{l}\text { Cycle time/plug flow residence time ratio } \\
\qquad\left(\frac{\tau P_{f} k}{\mu L^{2}}\right)\end{array}$ \\
\hline $\begin{array}{l}\text { Volume ratio } \\
\qquad\left(\frac{V_{R}}{A L}\right)\end{array}$ \\
\hline Dimensionless Parameters \\
\hline$f_{f}, f_{D}$, and $\left[Z_{f, i}\right] \quad$ for $i=1,2, \ldots, N$ \\
\hline
\end{tabular}

capacity is pressure-independent due to the linear sorption isotherm employed in the model, and when divided by $\epsilon$ results in a group that is more intuitively understood, the capacity enhancement factor,

$$
\left(\frac{\epsilon+\rho_{A} R T \alpha_{i}}{\epsilon}\right) \text { for } i=1,2, \ldots, N
$$

This factor relates the gas phase capacity for a component to the capacity of the combined gas solid mixture. Several of the PSR dimensionless groups are also common to single-bed rapid pressure swing adsorption.

Dimensional analysis clarifies the interactions between several variables. Interestingly, a shorter $P S R$ can increase adsorbent productivity (in terms of throughput per gram of adsorbent) without affecting conversion, separation, or catalyst productivity. For example, a change in the length of a PSR unit from $L$ to $L^{\prime}$ such that $L^{\prime}=1 / 2 L$, if accompanied by changes in $\tau$, $Q_{d}, \rho_{c}$, and $V_{R}\left(\tau^{\prime}=1 / 4 \tau, Q_{d}^{\prime}=2 Q_{d}, \rho_{a}^{\prime}=\rho_{a}, \rho_{c}^{\prime}=4 \rho_{c}, V_{R}^{\prime}=1 / 2 V_{R}\right)$, will result in the same conversion and separation with twice the throughput $\left(Q_{f}^{\prime}=2 Q_{f}, Q_{e}^{\prime}=2 Q_{e}\right)$. Adsorbent productivity $\left(Q_{f}^{\prime} L \rho_{a} / L^{\prime} \rho_{a}^{\prime} Q_{f}\right)$ will be quadrupled, while catalyst productivity will remain unchanged. This result is valid for any reaction order and for multiple reaction systems (even with mixed reaction orders). The dimensional analysis involving $k / \mu$ conducted previously (Vaporciyan and Kadlec, 1987), can also be repeated for reaction rate limited PSR performance.

These results must be interpreted with caution, as faster cycles, a shorter reactor, and a larger capacity will lead to higher flow rates, perhaps requiring the introduction of sorption rate limiting factors that have been neglected in the formation of the reaction rate limited model.

\section{Parameter determination}

One objective of the research was to determine the ability of the model to predict behavior of the actual process. Toward this end, values for various parameters relating to the experimental PSR apparatus were necessary: feed pressure, exhaust pressure, delivery flow rate, cycle time, feed fraction of the process period, delay fraction of the process period, feed composition, reaction rate law constants, reaction stoichiometry, amount of adsorbent, linear adsorption isotherm constants for each component, reflux void volume, gas viscosity, the length, temperature, cross-sectional area, and permeability of the packed bed, and the amount, loading, impregnation depth, and dispersion of the catalyst.

All operating parameters were controlled and directly measurable. The physical characteristics of the experimental PSR system are shown in Table 2 . These values were employed in the $\mathrm{CO}$ oxidation simulations. The gas viscosity was calculated assuming $100 \%$ nitrogen at the PSR temperature. The reflux void volume was measured by both water displacement and depressurization experiments. Bright field optical microscopy was employed to measure the impregnation depth of the platinum. The platinum dispersion on the alumina support was determined by hydrogen chemisorption. The platinum surface area was then calculated from Eq. $7 \mathrm{~b}$. Several reaction rate law parameters $\left(K_{1}, E, K_{2}\right.$, and $\left.H\right)$ were taken from Hegedus et al. (1977). A steady-state nitrogen purge through the packed bed 
Table 2. Physical Characteristics of Periodic Separating Reactor

\begin{tabular}{lc}
\hline Cross-sectional area & $3.45 \mathrm{~cm}^{2}$ \\
Reflux void volume & $50 . \mathrm{cm}^{2}$ \\
Packed bed length & $157.5 \mathrm{~cm}$ \\
Packed bed permeability & $48.6 \mathrm{~cm}^{2} \cdot \mathrm{cp} / \mathrm{atm} \cdot \mathrm{s}$ \\
Gas viscosity & $0.0228 \mathrm{cp}$ \\
Mass of catalyst & $229.6 \mathrm{~g}$ \\
$\Delta$ & $0.174 \mathrm{~mm}$ \\
$\delta$ & $0.163 \mathrm{~mm}$ \\
Platinum & $0.5 \mathrm{wt} . \%$ \\
Pt dispersion & $13.7 \%$ \\
$a$ & $10,040 \mathrm{~cm}^{2} \mathrm{Pt} / \mathrm{g} \mathrm{cat}$ \\
$K_{1}$ & $10^{8} \mathrm{~mol} \cdot \mathrm{K} / \mathrm{cm}^{2} \mathrm{Pt} \cdot \mathrm{s} \cdot \mathrm{atm}$ \\
$K_{2}$ & $5,484.51 / \mathrm{atm}$ \\
$E$ & $15,000 \mathrm{cal} / \mathrm{mol}$ \\
$H$ & $2,000 \mathrm{cal} / \mathrm{mol}$ \\
$M_{\text {ass of adsorbent }}$ & $143.1 \mathrm{~g}$ \\
$\gamma_{\mathrm{CO}}$ & 8.4 \\
$\gamma_{\mathrm{O}_{2}}$ & 1.9 \\
$\gamma_{\mathrm{CO}}$ & 51.4 \\
$\gamma_{\mathrm{N}_{2}}$ & 2.7 \\
\hline
\end{tabular}

was used to determine permeability. Packing void fraction was determined from $\mathrm{He}$ and $\mathrm{N}_{2}$ depressurization experiments. The linear adsorption parameters $\gamma_{\mathrm{CO}}, \gamma_{\mathrm{O}_{2}}, \gamma_{\mathrm{CO}_{2}}$, and $\gamma_{\mathrm{N}_{2}}$ were not available in the literature, for these conditions, and hence were determined by experiment. The model was used to determine the dimensionless capacity factors from three sets of RPSA experiments $\left(\mathrm{CO} / \mathrm{N}_{2}, \mathrm{O}_{2} / \mathrm{N}_{2}\right.$, and $\left.\mathrm{CO}_{2} / \mathrm{N}_{2}\right)$ performed at operating conditions otherwise similar to the PSR experiments. The values so determined seem consistent with extrapolated literature values. The equilibrium amount adsorbed on the alumina support is included in these values. The amount reversibly adsorbed on the Pt is not significant. The species involved have very different adsorption characteristics on the molecular sieve, as seen in Table 2. Separation of a mixture of $\mathrm{CO}, \mathrm{O}_{2}$, and $\mathrm{CO}_{2}$ from a bulk stream of $\mathrm{N}_{2}$ by PSA would result in the enrichment of $\mathrm{CO}_{2}$ and $\mathrm{CO}$ in the exhaust, and $\mathrm{O}_{2}$ enrichment in the delivery stream. In summary, all parameter values employed in the simulations were either measured directly or were determined from non-PSR experiments.

\section{Co Oxidation Experiments}

Many parameters affect the cyclic separation and conversion of the carbon monoxide-oxygen-nitrogen feed mixture. Of these, only the column configuration, catalyst, and the minimum exhaust pressure remained invariant throughout this investigation. The type and size of molecular sieve, the length of the delay and exhaust portions of the cycle, and the PSR temperature were established at suitable values by preliminary experimentation, and were not systematically varied. Base case operating parameters values are presented in Table 3 . These values were employed for all experimental results presented here unless otherwise noted. The effects of the percent oxygen in the feed, percent carbon monoxide in the feed, length of the feed portion of the cycle, the feed pressure, and the delivery flow rate are all illustrated and discussed. Based upon preliminary experiments and the conclusions drawn from the above experimental series and simulation results, the effects of the operating temperature and catalyst loading are discussed.

Clear distinctions among the interrelated terms separation factor, system throughput or capacity, delivery cut, recovery, extent of conversion, and production rate are essential for proper interpretation of PSR performance. The separation factor is defined as the ratio of the cycle average mole fractions of a component between the delivery and exhaust streams. Thus, for ratios above one, that component is enriched in the delivery stream. The system throughput or capacity is the cycle average flow rate $(\mathrm{mmol} / \mathrm{s})$ processed by the separating reactor. The delivery cut is the fraction of the throughput that exits the PSR at the delivery stream. Recovery refers to the percent of the total amount of a component exiting the PSR that is recovered in a particular stream. The production rate is defined as the total $\mathrm{mol} / \mathrm{s}$ of $\mathrm{CO}_{2}$ produced by the PSR, while the conversion is the percent of the feed carbon monoxide converted to $\mathrm{CO}_{2}$. (Product selectivity is defined for multiple reactions as the ratio of total conversion to the reference product divided by the total conversion to all other products).

For the experimental results presented here, the time required for attainment of cyclic steady state operation ranged from 18 to $48 \mathrm{~h}$ per experiment. The large adsorbent capacity for $\mathrm{CO}_{2}$, relative to the production rate of $\mathrm{CO}_{2}$, partially explains the long transients. The flow conditions reached an approximate cyclic steady state within several minutes. Runs with higher delivery

Table 3. Base Case Operating Parameter Corresponding to the Various Experiments

\begin{tabular}{|c|c|c|c|c|c|}
\hline & \multicolumn{4}{|c|}{ Reference Figure } & \multirow[b]{2}{*}{ Control Erro } \\
\hline & 3,4 & 5,6 & 7 & 8 & \\
\hline $\begin{array}{l}\text { Feed pressure, atm } \\
\text { Exhaust pressure, atm }\end{array}$ & $\begin{array}{l}1.68 \\
1.0\end{array}$ & $\begin{array}{l}1.68 \\
1.0\end{array}$ & $\begin{array}{l}1.68 \\
1.0\end{array}$ & $\begin{array}{c}1.68 \& 2.71 \\
1.0\end{array}$ & \pm 0.07 \\
\hline $\begin{array}{l}\text { Feed time, } s \\
\text { Delay time, s } \\
\text { Exhaust time, s }\end{array}$ & $\begin{array}{l}0.53 \\
1.47 \\
2.93\end{array}$ & $\begin{array}{l}0.63 \\
1.50 \\
2.93\end{array}$ & $\begin{array}{r}\text { varied } \\
1.47 \\
2.87\end{array}$ & $\begin{array}{l}0.53 \\
1.47 \\
2.93\end{array}$ & \pm 0.01 \\
\hline $\begin{array}{l}\text { Feed O, vol. \% } \\
\text { Feed CO, vol. \% } \\
\text { Balance } \mathrm{N}_{2}\end{array}$ & $\begin{array}{r}\text { varied } \\
1.60\end{array}$ & $\begin{array}{r}1.15 \\
\text { varied }\end{array}$ & $\begin{array}{l}2.67 \\
1.60\end{array}$ & $\begin{array}{l}2.67 \\
1.60\end{array}$ & $\begin{array}{l} \pm 0.08 \\
\pm 0.04\end{array}$ \\
\hline Temperature, $\mathrm{K}$ & 433 & 427 & 427 & 431 & \pm 2.8 \\
\hline $\begin{array}{l}\text { Delivery flow rate, } \mathrm{cm}^{3} / \mathrm{s} \\
\text { Delivery cut, } \%\end{array}$ & $\begin{array}{l}2.68 \\
13.1\end{array}$ & $\begin{array}{l}2.60 \\
12.6\end{array}$ & $\begin{array}{c}\text { varied } \\
12.7\end{array}$ & $\begin{array}{l}\text { varied } \\
\text { varied }\end{array}$ & $\begin{array}{l} \pm 0.06 \\
\pm 0.2\end{array}$ \\
\hline
\end{tabular}


flow rates, feed pressures, and feed compositions far from stoichiometric required less time to reach cyclic steady state. The cyclic steady state for the base case operating parameters was approached from several different initial bed conditions. Bifurcations were not noted, as parameter ranges were traversed in both increasing and decreasing directions. Only a single stable periodic attractor was observed (within experimental errors) for each set of operating parameters; however, concentration measurements were averaged over many cycles.

The effect of the percent oxygen in the feed upon the performance of the PSR is shown in Figures 3 and 4 . As the oxygen in the feed is increased, conversion increases linearly up to $\sim 60 \%$ $\mathrm{CO}$ conversion (at $1.5 \%$ oxygen in the feed), where it remains unchanged. The PSR separation performance changes drastically as the feed oxygen is varied, Figure 3 . The most intriguing feature is the occurrence of separation reversals. At low feed oxygen concentrations, oxygen is enriched in the exhaust stream while carbon dioxide is concentrated in the delivery. As the feed oxygen is increased, separations reverse, with carbon dioxide enriched in the exhaust and oxygen concentrated in the delivery stream. Another feature is that at low oxygen feed concentrations only $\mathrm{CO}$ and carbon dioxide are present in the delivery stream, while at higher feed oxygen concentrations only carbon dioxide and oxygen are present in the delivery stream. Interestingly, the disappearance of $\mathrm{CO}$ from the delivery stream coincides with the maximum in the $\mathrm{CO}_{2}$ concentration, Figure 4. All three reaction components are present in the exhaust stream over the entire range of feed oxygen concentrations. Overall, simulation results properly predict the oxygen experimental series.

An understanding of the coupling between the reaction and separation performance of the PSR can be obtained by examining simulation results for the bed interior. For low conversions most of the $\mathrm{CO}_{2}$ is produced near the delivery end of the PSR. At the feed oxygen concentration for which $\mathrm{CO}$ is no longer present in the delivery stream, a sharp reaction front develops at the delivery end of the packed bed. A reaction front is expected from the form of the rate law for CO oxidation on platinum, Eq. $7 \mathrm{a}$, due to the change from a positive reaction order in $\mathrm{CO}$ to negative reaction order at high carbon monoxide concentrations. Even though the adsorption of carbon dioxide is extensive, the product formed so near the delivery stream cannot be com-

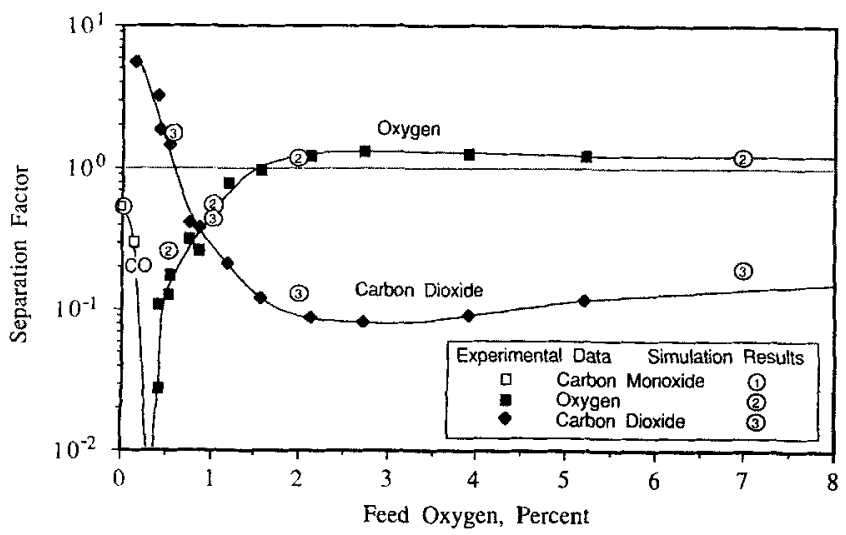

Figure 3. Separation performance as a function of feed oxygen concentration.

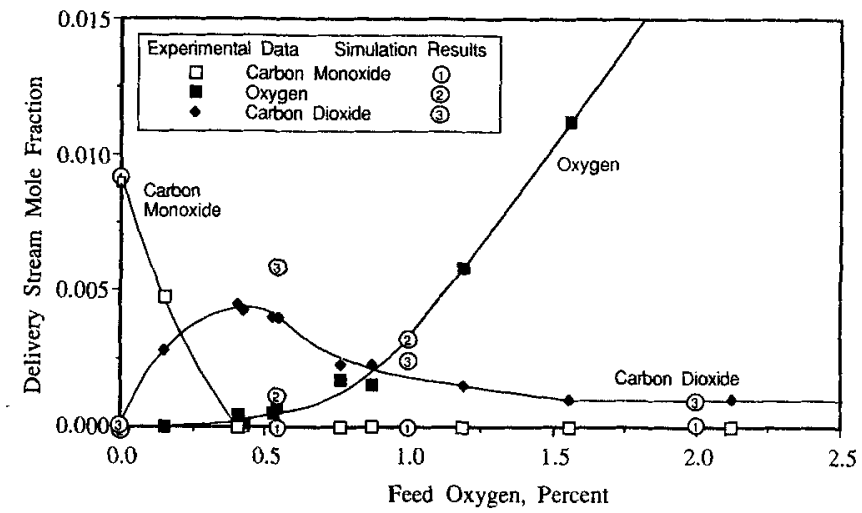

Figure 4. Effect of feed oxygen concentration on delivery stream composition.

pletely drawn toward the exhaust stream, and thus is enriched in the delivery stream. As the feed oxygen concentration is increased further, the reaction front moves toward the feed end of the bed, thus the decrease in the $\mathrm{CO}_{2}$ concentration in the delivery stream, and finally the separation reversal. At oxygen concentrations above $-1.5 \%$, the reaction front is within onetenth the total bed length from the feed end.

Oxygen is enriched in the exhaust stream at low feed oxygen concentrations due to a greater consumption of $\mathrm{O}_{2}$ down the length of the packed bed rather than enrichment due to RPSA. At feed oxygen concentration above $\sim 0.4 \%$, carbon monoxide is absent from the delivery stream due to the complete consumption of any $\mathrm{CO}$ that reaches the reaction front.

The effect of the concentration of carbon monoxide in the feed stream upon the PSR performance is shown in Figure 5. Conversion within both steady-state and periodic separating reactors decreases with increasing inlet $\mathrm{CO}$ due to the inhibition effect of $\mathrm{CO}$ on the reaction rate. The inlet concentration of $\mathrm{CO}$ at which the maximum in the steady-state oxidation rate occurs is independent of the feed $\mathrm{O}_{2}$ and is simply equal to [1/ $\left.K_{2} \exp (H / R T)=1.76 \times 10^{-5} \mathrm{~atm}\right]$. Thus for the majority of the range of feed $\mathrm{CO}$ concentrations investigated reaction is inhibited by $\mathrm{CO}$.

PSR and steady-state operation are compared at equal feed



Figure 5. Carbon dioxide production rate vs. feed carbon monoxide concentration. 
concentrations and cycle average flow rates with the steadystate feed pressure equal to the PSR high feed pressure. Although the production rate of $\mathrm{CO}_{2}$ can be greater during steady-state plug-flow operation than PSR, the locations of the maxima are different. The maximum in the $\mathrm{CO}_{2}$ production rate for steady-state operation is narrower and occurs at an inlet mixture that is oxygen-rich. The maximum for PSR operation is broader and is centered at the stoichiometric ratio for the feed mixture. Over a wide range of inlet $\mathrm{CO}$ concentrations, PSR operation results in over twice the $\mathrm{CO}_{2}$ production of the plug-flow alternative.

The difference between the steady-state and PSR performance is due to three effects. Foremost are the effects of separation. At high $(\sim 1.6 \%)$ feed concentrations of $\mathrm{CO}$, removal of excess CO by RPSA in the exhaust stream results in greater oxidation rates throughout the rest of the PSR. The net result of such a separation is greater conversions than are possible within a steady-state plug-flow reactor. At lower feed $\mathrm{CO}$ concentrations, oxidation rates are already sufficient to result in high conversions and thus any removal of $\mathrm{CO}$ in the exhaust stream reduces the overall conversion.

The second phenomenon that affects the oxidation rate in a PSR is caused by adsorption of the reactant. At cyclic steady state the cycle average $\mathrm{CO}$ fed to the PSR either reacts or exits the reactor. Local removal of $\mathrm{CO}$ from the gas phase by the adsorbent during the feed part of the cycle results in higher local oxidation rates because of the inhibition effect of the reactant The subsequent desorption of $\mathrm{CO}$ slows the oxidation rate during the exhaust portion of the cycle. The opposing effects do not cancel because $\mathrm{CO}$ lost to reaction during the feed portion of the cycle will not be present to inhibit the reaction during the exhaust portion of the cycle. The net effect is an increase in the overall local oxidation rate.

The third phenomenon that also plays a role in the conversionfeed $\mathrm{CO}$ performance difference is residence time distribution (RTD) differences. For a given level of conversion, the mean residence time of the steady-state plug-flow reactor is independent of the feed CO concentration, however for the PSR there is a dependence. Within a PSR, mean residence time can be altered by the adsorption of the reactant (Vaporciyan and Kadlec, 1987), due to the existence of two exit streams. In this case additional $\mathrm{CO}$ in the feed results in deeper penetration of the adsorption front for $\mathrm{CO}$ (even if conversion was not altered). In turn, the average residence time for $\mathrm{CO}$ is increased. Thus, as the inlet concentration of $\mathrm{CO}$ to the PSR is increased, the average residence time for the reactant increases.

The separation performance of the PSR as a function of the feed CO concentration is shown in Figures $6 \mathrm{a}$ and $6 \mathrm{~b}$. Separation reversals for oxygen and carbon dioxide occur. Three regions can be identified. At low $\mathrm{CO}$ feed concentration, only oxygen is present in the delivery stream. At intermediate $\mathrm{CO}$ feed concentrations, both carbon dioxide and oxygen are present in the delivery stream. At higher feed CO concentrations, all three reaction components are present in the delivery stream. All three reaction components are present in the exhaust stream for the entire range of feed $\mathrm{CO}$ concentrations.

The presence of a reaction front again explains the results. At low feed concentrations of $\mathrm{CO}$, the reaction front exists near the feed end of the packed bed, thus the $\mathrm{CO}_{2}$ produced is enriched in the exhaust stream. As the feed concentration of $\mathrm{CO}$ is

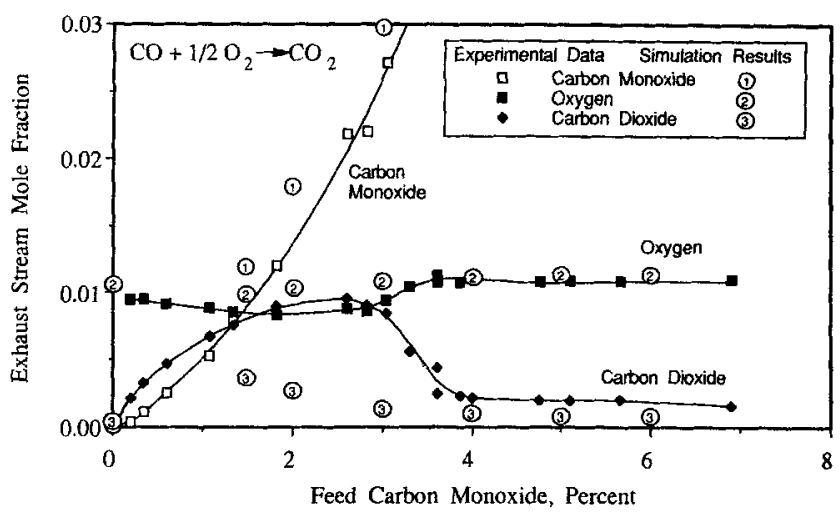

Figure 6a. Effect of feed carbon monoxide concentration on exhaust stream composition.

increased, the front moves further into the packed bed, and thus $\mathrm{CO}_{2}$ in the delivery stream increases while the $\mathrm{CO}_{2}$ in the exhaust stream goes through a maximum. At a feed $\mathrm{CO}$ concentration of about $4.0 \%$ the reaction front reaches the delivery end of the bed. At higher feed CO concentrations the front exits the bed and thus $\mathrm{CO}$ is found in the delivery stream.

Additional data indicating the existence of the sharp reaction front were collected from the sampling ports down the length of the PSR packed bed. Cyclic steady state temperature profiles also indicated the existence of the reaction front. Temperature variations during a cycle were within one degree, while temperature profiles down the length of the packed bed varied by five degrees. For some experiments in which the reaction front existed near one of the thermocouples, that temperature would be statistically greater than all other thermocouple measurements.

Most $\mathrm{CO}$ feed series simulations did not reach cyclic steady state (within $0.05 \%$ ) within an affordable computation time. One consequence of the solution method is that movement of the front is in discrete increments. Therefore, when the reaction front is very near the feed end of the PSR simulation results would be expected to deviate furthest from experimental results. Such discrepancy was found to be the case. Although the simulation results do not fit the experimental data as well as in the oxygen feed series, the model shows the reaction front phenomena and its movement as the feed $\mathrm{CO}$ concentration is increased.



Figure 6b. Effect of feed carbon monoxide concentration on delivery stream composition. 
Regarding the quantitative ability of the model, a critical assumption implicitly made is that the reaction rate law is valid during periodic operation. The assumption seems correct when examining the oxygen series; however, this simply may not be the case for the cycle times and range of inlet $\mathrm{CO}$ concentrations employed for the feed $\mathrm{CO}$ series. Another possibility is the employment of linear isotherms in the model. Besides the possibility of a different form of sorption isotherm, adsorption of carbon dioxide on the molecular sieve is so strong that competitive adsorption may occur. Finally, values for $K_{2}, E$, and $H$ were taken from the literature and could be different for the particular batch of catalyst employed. One important conclusion regarding the model is that all observed phenomena for PSR CO oxidation can be qualitatively (and to a good extent quantitatively) predicted with a model that employs a large set of simplifying assumptions.

The production rate of carbon dioxide as a function of the feed portion of the process cycle is shown in Figure 7. The length of the delay and exhaust portions of the process cycle have been kept constant. A production rate maximum exists for two reasons. First, the cycle average feed flow rate response to the feed time has a maximum near $3.5 \mathrm{~s}$ (a fluid flow phenomenon). Secondly conversion increases as the feed time is increased and reaches a plateau of $65 \%$ at $2.5 \mathrm{~s}$, thus causing the production maximum at $3 \mathrm{~s}$.

The effect of the delivery cut upon the delivery mole fractions was examined at two feed pressures. An upper limit exists on the delivery cut that can be obtained while maintaining a constant delivery stream flow rate. Higher delivery cuts are possible at higher feed pressures. Oxygen concentration in the delivery stream decreases as the delivery cut is increased. This result is due to the separation performance of the PSR alone, as the conversion achieved in the PSR remains constant at $\sim 58 \%$ throughout the experimental series. Rapid pressure swing adsorption also exhibits such behavior as the delivery cut is increased (Kowler and Kadlec, 1972).

Although carbon monoxide was not detected in the delivery stream as the delivery cut was varied, breakthrough of carbon dioxide was seen, Figure 8. As would be expected from RPSA

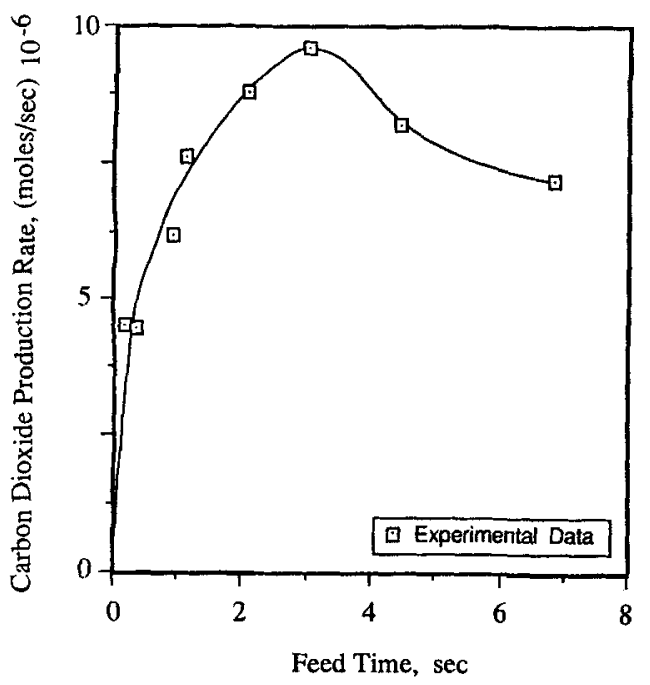

Figure 7. Production rate of carbon dioxide vs. duration of high-pressure feed.

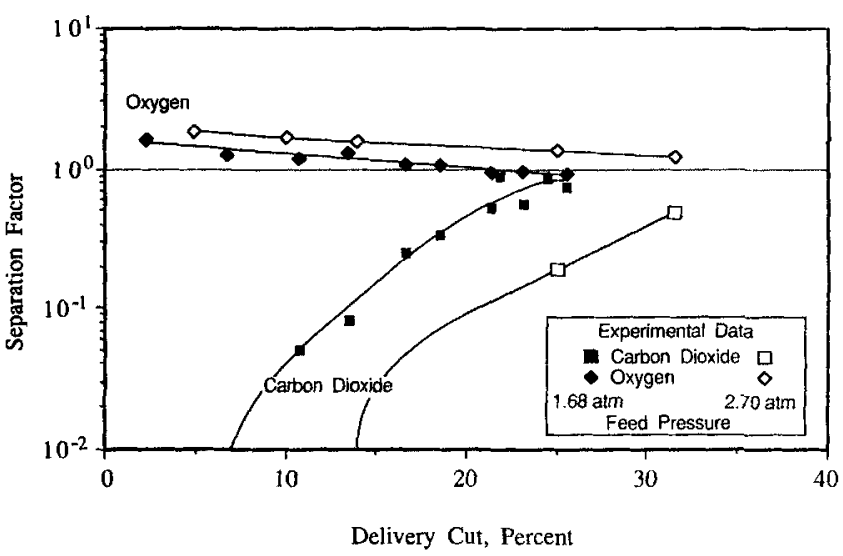

Figure 8. Effect of delivery cut and feed pressure on component separation factors.

theory, a higher degree of separation is achieved at lower delivery cuts and at the higher pressure. The breakthrough of $\mathrm{CO}_{2}$ occurs at a higher delivery cut at the higher feed pressure. In this case, the behavior is a result of both separation and reaction performance. At higher delivery cuts the reaction front is situated closer to the delivery stream. Coupled with the poorer separation achieved at the higher delivery cuts, $\mathrm{CO}_{2}$ breakthrough is observed.

At this point the effects of temperature and catalyst loading can also be examined. The separating reactor was designed to provide separation with very limited reaction at room temperature. The degree of reaction and separation achieved covers the broadest range as temperature or catalyst loadings are varied. With no catalyst present or at very low tempertaures, no conversion is achieved, and thus the PSR operates as RPSA with the separation of the reactants from the diluent. At low catalyst loadings or low temperatures the reaction front phenomenon is observed near the delivery end of the bed and moves toward the feed end as catalyst loading or PSR temperature is increased. At the extreme, high catalyst loadings or high temperatures result in the instantaneous complete conversion of the limiting reactant upon being fed to the PSR. The effect of these parameters upon the separation component of PSR is not the same. Whereas catalyst loading has no effect upon the adsorbent capacity, higher temperatures reduce the capacity of the adsorbent.

\section{Other Reaction Systems}

Through simulation, numerous irreversible, reversible, and multiple reaction systems were investigated. The physical parameters that varied included reaction stoichiometry, adsorbent coefficients, and PSR Damköhler number. Design variables included feed pressure, delivery flow rate, cycle period, and the feed and delay fractions of the process period. Physical characteristics of the simulated PSR were similar to the experimental PSR. Preliminary runs led to the base case operating conditions shown in Table 4. These parameter values were employed in all runs unless otherwise stated. The range of capacity enhancement factors employed is conservative, as the capacity enhancement factors for the experimental system ranged from $\sim 3$ for $\mathrm{O}_{2}$ to $\sim 82$ for $\mathrm{CO}_{2}$. Results were evaluated based upon degree of separation, system throughput, product selectivity, extent of 
Table 4. Base Case Values for Dimensionless Groups and Varied Parameters



conversion, and component recovery in the two product streams, as previously defined.

\section{Irreversible reactions}

Three irreversible reaction systems were investigated employing elementary homogeneous rate laws: $A \rightarrow 1 / 2 B+1 / 2 C, A \rightarrow B$, and $C \leftarrow A \rightarrow B$. As the Damköhler number is increased from $\sim 10^{-2}$ to $10^{2}$, conversion increases from $\sim 2$ to $\sim 99 \%$. For a given value of the PSR Damköhler number, conversions are lower for reaction systems in which the reactant is adsorbed (relative to the diluent). This result is a consequence of the reactant residence time distribution and local adsorption. Pressure swing adsorption theory predicts that when a component is adsorbed it will be enriched in the exhaust stream, otherwise enrichment will occur in the delivery stream. Due to the fact that component $A$ is fed at the exhaust end of the bed, the average residence time for the reactant will be shorter when adsorption takes place. The shorter residence time therefore leads to lower conversions. Reduced gas phase concentration of the reactant due to local adsorption (on the adsorbent thus nonreactive) also leads to lower conversions.

The separation performance of a PSR for one irreversible reaction system is shown in Figure 9, which is typical of those investigated. Several features of such systems became evident. In general, physical adsorption properties may not exert the same effect in PSR as in PSA. At low values of the PSR Damköhler number, the conversion achieved is small, thus negligible amounts of products exist and the separation of the reactant is as

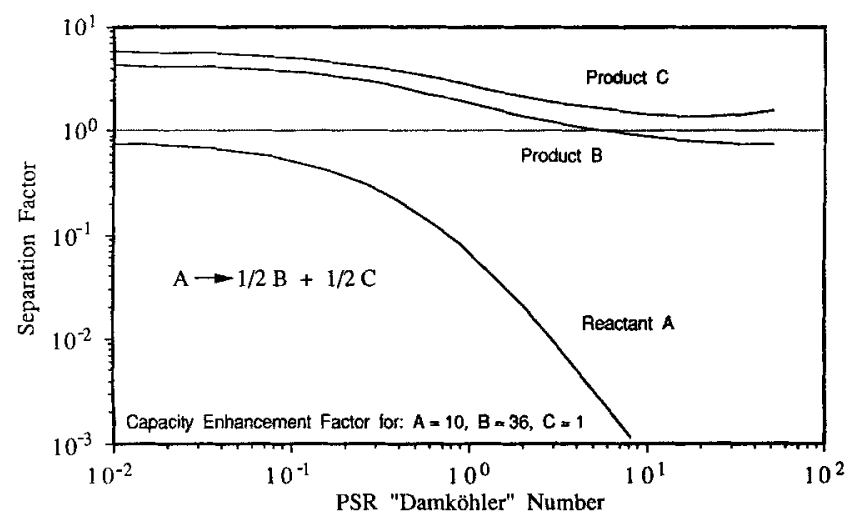

Figure 9. Separation factors vs. PSR Damköhler number for the reaction $A \rightarrow 1 / 2 B+1 / 2 C$. in a RPSA process. As the PSR Damkohler number is increased, the total amount of reactant $A$ decreases while additional product $B$ and $C$ are produced. At this intermediate level of Damköhler number significant amounts of product are produced very near the delivery stream because of the low velocities (long holding times) near the delivery stream end of the PSR unit. This in turn results in the inability of the PSR unit to enrich the preferentially adsorbed product $B$ in the exhaust stream. At higher values of the PSR Damköhler number the products are produced nearer the feed end of the PSR unit and thus the adsorbed product $B$ undergoes a separation reversal and is found enriched in the exhaust stream. Finally, at very large Damköhler numbers complete reactant conversion is instantaneous and thus the PSR unit operates as a RPSA process being fed the two products with diluent. The manner by which the Damköhler number effects the separation reversal of product $B$ is similar to how oxygen affects the separation reversal of carbon dioxide in the PSR experiments.

Concentration histories at different lengths into the PSR are given in Figure 10 for the reaction $A \rightarrow 1 / 2 B+1 / 2 C$. Bed pressures increase during the feed portion of the period and decrease during the exhaust portion. The product $B$ is removed from the gas phase as it is produced during the feed portion of the cycle and then released during the exhaust portion of the cycle. Enrichment occurs toward the feed end of the PSR. The oppo-
Mole Fraction A

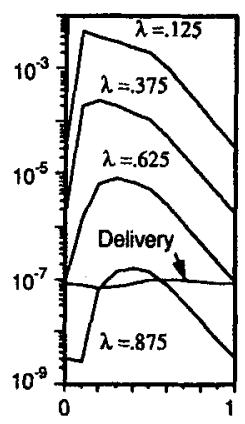

Mole Fraction B

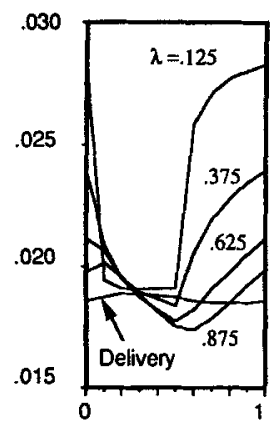

Mole Fraction $\mathrm{C}$

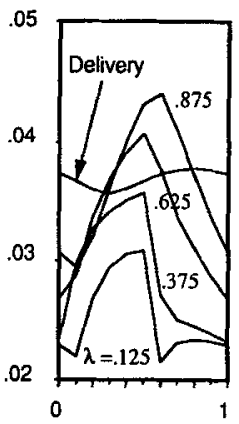

Dimensionless Cycle Time

Figure 10. Concentration histories of components at varlous distances into PSR for the reaction $A \rightarrow$ $1 / 2 B+1 / 2 C$.

Damköhler number $=52.3$; capacity enhancement factor for $A=$ $10, B-36, C=1$. 
site effect is observed for product $C$. Due to the lack of adsorption, accumulation is in the gas phase during the feed portion of the cycle and enrichment occurs toward the delivery end of the PSR.

The concentration profiles are somewhat different for the reactant. The step change in the inlet concentration of $A$ is increasingly damped at greater distances into the bed due to adsorption, reaction, and pressure transients. The very fast reaction rates for this case result in decreasing reactant concentration during the exhaust portion of the cycle. Reaction rates coupled with the RPSA separation result in almost four orders of magnitude difference in the concentration of $A$ from the exhaust to delivery end of the packed bed.

\section{Reversible reactions}

Three reversible reactions systems are investigated: $A=$ $1 / 2 B+1 / 2 C, A=B$, and $C \leftrightharpoons A=B$. The conversion-Damköhler number performance of the various cases may be placed into two groups, those in which the reactant is adsorbed and those in which it is not adsorbed. At the two extremes, performance of the two groups is the same; for very low Damköhler numbers there is no conversion while at very large Damköhler values performance is predicted by equilibrium-limited PSR (EPSR) theory. For all three reaction systems, the dimensionless reaction constant is pressure-independent and thus EPSR theory predicts the same level of conversion achievable in a conventional reactor at reaction equilibrium (Vaporciyan and Kadlec, 1987). Conversion performance between these two limits is different for the two groups.

First, as for irreversible reactions, conversions are greater for reaction systems in which the reactant is not adsorbed. Second, if the reactant is not adsorbed, the conversion goes through a local maximum, the value of which is greater than the equilibrium conversion possible in a conventional reactor. The first result is not only a consequence of the different mean reactant residence time, (as was the case for irreversible reaction systems), but also of the effect of adsorption on the reaction equilibrium. The conversion enhancement is solely a consequence of the reaction equilibrium shift.

The shift in reaction equilibrium phenomena can be explained by invoking LeChatelier's principle. Product $B$ in all of the systems investigated is preferentially adsorbed; thus as a reaction proceeds, product is removed from the gas phase accumulating on the adsorbent. This shifts the reaction equilibrium further toward the products. Those systems in which the reactant is absorbed would tend to experience a shift of the reaction equilibrium back toward the reactant. For the reaction system $A=$ $1 / 2 B+1 / 2 C$, separation of the two products from one another also inhibits the reverse reaction.

The separation and subsequent reaction equilibrium shift possible in a PSR unit can cause favorable selectivity changes in multiple reaction systems. The conversion-Damköhler number performance for one parallel reversible system investigated $(C \leftrightharpoons A \leftrightharpoons B)$ is shown in Figure 11. With $B$ preferentially adsorbed, and equal rate and equilibrium constants, selectivity toward component $B$ is enhanced compared to conventional reactor operation, which can never show selectivity toward either product (i.e., a selectivity of 1). Comparison is made at equal feed concentrations and cycle average flow rates with the steady-state feed pressure equal to the PSR high feed pressure. Selectivity goes through a local maximum as PSR conversion

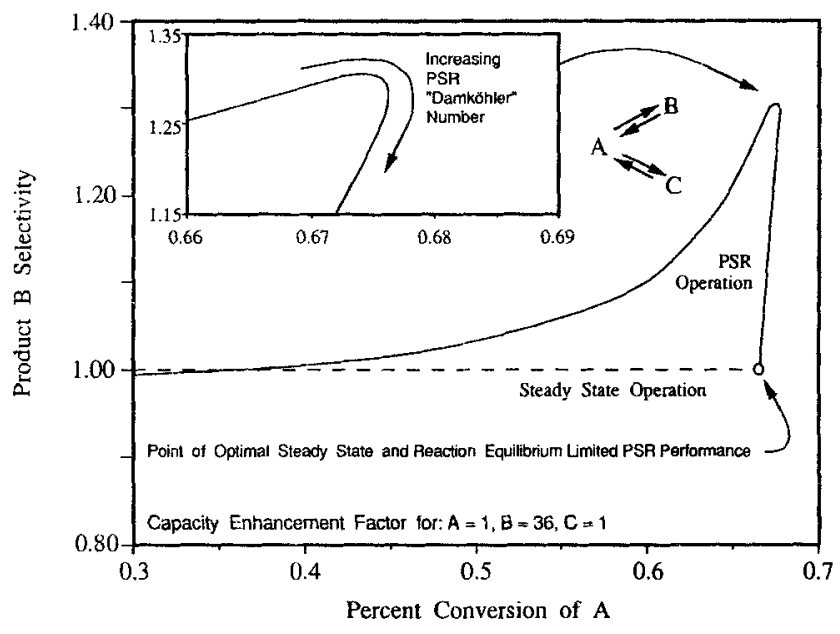

Figure 11. Selectivity toward product $B$ as a function of total conversion of reactant $A$.

increases. There is a balance between the separation and reaction components of the PSR, which results in the optimal selectivity of the reaction toward the product $B$. The greatest selectivity is achieved when the PSR conversion surpasses the equilibrium conversion possible in a conventional reactor $(66.67 \%)$. In addition, for the upper range of possible conversions, two levels of selectivity are possible depending upon the particular PSR Damköhler number. As reaction rates increase, selectivity performance will reach the limit predicted by instantaneous local reaction equilibrium.

\section{Input wave form}

As discussed earlier there are many design parameters for the periodic separating reactor, even after limiting the type of PSA and input wave form employed. In addition to the parameters investigated experimentally, the dimensionless pressure ratio, delivery flow rate, total cycle time, feed fraction of the period, and delay fraction of the period were investigated for a parallel reversible reaction system with equal rate and equilibrium constants. Once again, the best a steady-state plug-flow or CSTR system could do is $66.67 \%$ conversion with no selectivity toward either product.

Larger dimensioniess pressure ratios $\left(P_{f} / P_{e}\right)$ increase selectivity, total conversion, and separation. For example, increasing $P_{f} / P_{e}$ from 1.68 to 6.72 results in an increase of total conversion from 67 to $70 \%$ and product $B$ selectivity from 1.3 to 1.6 .

The effect of the total cycle period upon the selectivity within a PSR is shown in Figure 12. There exists a cycle period range within which the device may operate. If the cycle period is too short, the bed pressure attenuation is so great that the delivery flow rate cannot be realized. At the other end of the period range, long cycle times lead to long exhaust times and thus also result in a system incapable of sustaining a set delivery flow rate. Product $B$ selectivity exhibits a local maximum within the realizable range of periods. The location of the maximum occurs at the same frequency at which the separation performance exhibits a maximum. Such a maximum in the separation performance of an experimental RPSA unit has been observed (Kowler and Kadlec, 1972). Conversion of the reactant remained invariant at approximately $67 \%$ over the operating frequency range.

The effect of the fraction of the period that the feed is intro- 


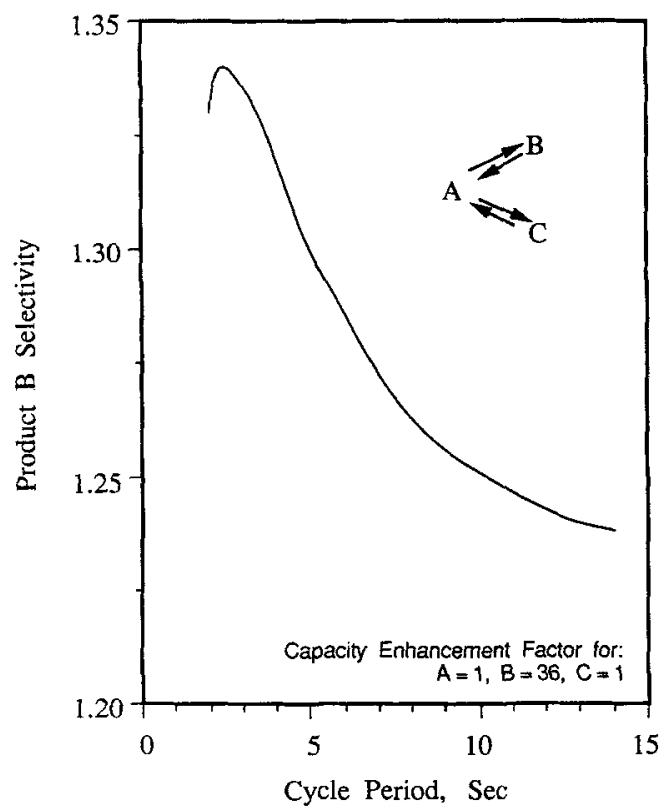

Figure 12. Effect of frequency on product $B$ selectivity.

duced into the PSR upon the selectivity is shown in Figure 13. Two limits on the operating feed fraction exist. If the feed fraction is too short, not enough gas is fed to the PSR to maintain the set delivery flow rate. At the other limit, a feed fraction of one is equivalent to steady-state plug-flow operation with no exhaust stream and therefore no separation or selectivity enhancement.

Product $B$ selectivity exhibits a local maximum that occurs at large values of the feed fraction. During the exhaust portion of the cycle reaction rates for the reverse reaction of $B$ to $A$ are greatest due to the high concentration of $B$ in the gas phase as it is released from the adsorbent. An excessive exhaust time would

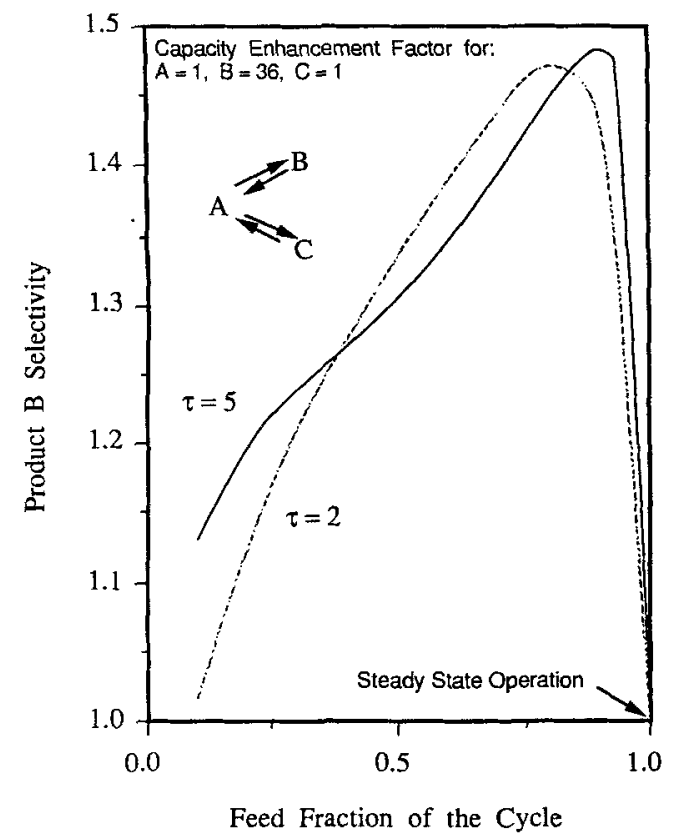

Figure 13. Effect of cycle feed fraction and period on product $B$ selectivity. allow significant amounts of the product to convert back to the reactant $A$. The effects on separation are weak, with separation factors varying less than $5 \%$ between feed fractions of 0.1 and 0.9 over the realizable range of feed fractions. The effect of the feed fraction of the process cycle upon the dimensionless feed and exhaust flow rates is qualitatively similar to that simulated for an equilibrium-limited PSR (Vaporciyan and Kadlec, 1987) and to that observed experimentally for rapid single-bed PSA (Kowler and Kadlec, 1972).

The effect of increasing the delay portion of the process cycle is to increase the recovery of all components in the delivery stream. Although conversions and separations are basically invariant through the range of feasible delay fractions, percent recoveries of all three reaction components almost double.

\section{Conclusions}

A new unit operation that effects both continuous gas phase reaction and separation has been explored. The periodic separation reactor (PSR) utilizes a rapid pressure swing cycle in a catalytic/adsorbent bed. Feed sequences studied were those of rapid-cycle, single-bed pressure swing adsorption (RPSA). The device was built and the effects of various process variables were experimentally investigated for the oxidation of carbon monoxide over a mixture of platinum-supported catalyst and $5 \AA$ molecular sieve adsorbent. A general multicomponent reaction rate limited equilibrium sorption model was formulated and solved for a variety of irreversible and reversible reactions.

The presence of a chemical irreversible reaction is shown to greatly enhance the separation achievable by RPSA alone. For reversible reaction systems separation performance is within the same order of magnitude as sorption equilibrium RPSA. Unusual features of operation, such as separation reversals, were also predicted and observed. In general, the capacity performance for the PSR system is the same order of magnitude as sorption equilibrium RPSA.

Any ir reversible reaction system will exhibit two limits within the PSR system investigated: that of pure RPSA at one end, in which reactant separation is achieved with no reaction, and instantaneous complete conversion as the other limit, in which product separation occurs as in RPSA. A PSR system with finite rates of reaction shows maxima between the two limiting cases. Although $\mathrm{CO}$ oxidation might not show the true potential of PSR operation, for a wide range of inlet $\mathrm{CO} / \mathrm{O}_{2}$ ratios $\mathrm{CO}_{2}$ production could be increased up to two times over steady-state plug-flow reactor operation, while providing a recycle stream without phase change or extractive procedures. Conversion enhancements are possible for CO oxidation within the PSR investigated due to the inhibition of the reaction rate by $\mathrm{CO}$.

Conversions greater than equilibirum were predicted for a reversible reaction system at reaction rates between those resulting in RPSA and equilibrium-limited PSR (EPSR) performance, without the inherent limitations of chromatography reactors (Chu and Tsang, 1971). For systems with multiple reversible reactions, selectivity and conversion improvements over steady-state operation can be achieved. In addition, the perturbed reaction equilibrium model (Vaporciyan and Kadlec, 1987, has been shown to represent a limiting case for reversible reaction systems that is conservative in its predictive nature.

For reaction rate limited PSR performance, results were interpreted by considering reaction phenomena (residence time distributions, the nature of the reaction rate law, and equilib- 
rium shifts) coupled to conventional RPSA behavior. Although separation reversals were induced by manipulation of any one of a variety of parameters, the common underlying cause is the opposing concentration gradients created by the reaction and separation components of the process. Dimensional analysis also yields information about the interaction of different variables.

Interestingly, it has been determined that a conventional reaction rate law, derived from steady-state data, can be used qualitatively, and to a degree quantitatively, to predict cyclic steady state PSR performance within the range of operating conditions for which model and experiments were compared. The modeling approach properly reveals basic PSR phenomena (including separation reversals) without unduly complicating interpretation of the underlying reasons for such behavior. Incorporation of a fundamental reaction mechanism into the PSR model should lead to a more complete understanding of the effects of the periodic processing scheme.

It is significant that the periodic process has been shown to improve performance over the steady-state plug-flow reactor alternative and the underlying PSA process, for a variety of reactions. A PSR can operate in regions unattainable through other means and has been shown to provide recycle without phase change or extractive procedures. No attempt was made to optimize performance, but such study might further extend the range of applicability for the dynamic process. Joined with the multibed and rotational versions, an entirely new set of separating-reactor processes emerges for potential use by industry.

\section{Notation}

$A=$ cross-sectional area of periodic separating reactor, $\mathrm{cm}^{2}$

$a=$ accessible platinum surface area density, $\mathrm{cm}^{2} \mathrm{Pt} / \mathrm{g}$ cat

$C=$ fractional catalyst Joading, weight

$D=$ fractional dispersion

$E=$ activation energy, cal $/ \mathrm{mol}$

EPSR - Reaction-equilibrium-limited Periodic Separating Reactor

$f=$ fraction of the process period.

$f=$ a function

$H=$ heat of carbon monoxide adsorption over platinum, cal $/ \mathrm{mol}$

$J=$ total number of reactions

$K_{1}=$ kinetic preexponential, $\mathrm{mol} \cdot \mathrm{K} / \mathrm{cm}^{2} \mathrm{Pt} \cdot \mathrm{s} \cdot \mathrm{atm}^{2}$

$K_{2}=$ preexponential of carbon monoxide adsorption term, $1 / \mathrm{atm}$

$K_{e q}=$ reaction equilibrium constant, $\mathrm{atm}^{\Delta \nu}$

$k=$ permeability of packed bed, $\mathrm{g} \cdot \mathrm{cm} / \mathrm{atm} \cdot \mathrm{s}^{2}$

$\hat{\epsilon}=$ reaction rate constant, $1^{(\beta-1)} / \mathrm{s} \cdot \mathrm{mmol}^{(\beta-1)}$

$\overline{\boldsymbol{k}}=$ set of all reaction rate law parameters for a given reaction

$L=$ length of packed bed, $\mathrm{cm}$

$N=$ total number of components in system

$P=$ pressure, atm

PSA $=$ Pressure Swing Adsorption

PSR $=$ Periodic Separating Reactor

$Q=$ cycle average molar flow rate, $\mathrm{mmol} / \mathrm{s}$

$R=$ ideal gas constant

RPSA = Rapid Cycle Pressure Swing Adsorption

$r=$ intrinsic reaction $\mathrm{rate}, \mathrm{mol} / \mathrm{g}$ cat $\cdot \mathrm{sec}$

$S=$ arbitrary reaction component

$T=$ absolute temperature, $\mathrm{K}$

$t=$ time into cycle, $\mathrm{s}$

$V=$ volume, $\mathrm{cm}^{3}$

$x=$ distance down packed column from feed point, $\mathrm{cm}$

$Z$ - dimensionless pressure, $P / P_{f}$

\section{Greek letters}

$\alpha=$ constant in linear adsorption isotherm, $\mathrm{mmol} / \mathrm{atm} \cdot \mathrm{g} \mathrm{ad}$ sorbed

$\beta=$ reaction rate order in a forward reaction expression

$\gamma=$ dimensionless bed capacity, Eq. 2

$\Delta=$ catalyst support radius, $\mathrm{cm}$
$\Delta \nu=$ net change in total moles due to reaction

$\delta=$ inner radius of eggshell catalyst penetration, $\mathrm{cm}$

$\epsilon=$ porosity of packed bed

$\theta=$ dimensionless time, $t / \tau$

$\lambda=$ dimensionless length down packed bed, $x / L$

$\mu=$ average viscosity of gases, $\mathrm{g} / \mathrm{cm} \cdot \mathrm{s}$

$\rho=$ density of adsorbent or catalyst, $\mathrm{g} / \mathrm{cm}^{3}$

$\sigma=$ reaction rate order in a reverse reaction expression

$\nu=$ stoichiometric coefficient of subscripted chemical component

$\tau=$ cycle period, $\mathrm{s}$

\section{Subscripts}

$A=$ adsorbent

$C=$ catalyst

$D=$ delay part of cycle

$d$ - delivery stream

$e=$ exhaust stream or part of cycle

$f=$ feed stream or part of cycle

$i=i$ th chemical component of $N$ present

$j=j$ th reaction of total present

$l=$ reference component for particular reaction

$\min =$ minimum dimensionless pressure, $P_{e} / P_{f}$

$R=$ relating to reflux void volume

\section{Superscripts}

' = same parameter set

\section{Literature Cited}

Bailey, J. E., "Periodic Phenomena in Chemical Reactor Theory," Chemical Reactor Theory, L. Lapidus, N. R. Amundson, eds., Prentice-Hall, Englewood Cliffs, NJ (1977).

Barshad, Y., "Improving the Performance of Catalytic Reactors by Periodic Operation," Ph.D. Thesis, Univ. Michigan, Ann Arbor (1985).

Chu, C., and L. C. Tsang, "Behavior of a Chromatographic Reactor," Ind. Eng. Chem. Process Des. Dev., 10, 47 (1971).

Hamer, J. W., and D. E. Cormack, "Influence of Oscillating External Pressure on Gas-Phase Reactions in Porous Catalysts," Chem. Eng. Sci., 33, 935 (1978).

Hegedus, L. L., S. H. Oh, and K. Baron, "Multiple Steady States in an Isothermal, Integral Reactor: The Catalytic Oxidation of Carbon Monoxide over Platinum-Alumina," AIChE J. 23, 632 (1977).

Horn, F. J. M., and R. C. Lin, "Periodic Processes: A Variational Approach," Ind. Eng. Chem. Process Des. Dev., 6, 21 (1967).

Kowler, D. E., and R. H. Kadlec, "The Optimal Control of a Periodic Adsorber. I: Experiment, II: Theory," $A I C h E J ., 18,1207$ (1972).

Lee, S. Y., and R. Aris, "The Distribution of Active Ingredients in Supported Catalysts Prepared by Impregnation," Catal. Rev. Sci. Eng. 27, 207 (1985).

Oh, S. H., J. C. Cavendish, and L. L. Hegedus, "Mathematical Modeling of Catalytic Converter Lightoff: Single-Pellet Studies," AIChE $J ., 26,935$ (1980).

Razon, L. F., and R. A. Schmitz, "Intrinsically Unstable Behavior During the Oxidation of Carbon Monoxide on Platinum," Catal. Rev. Sci. Eng., 28(1), 89 (1986).

Schrodt, V. N., "Unsteady-State Processing," Ind. Eng. Chem., 59(6), 58 (1967).

Turnock, P. H., and R. H. Kadlec, "Separation of Nitrogen and Methane via Periodic Adsorption," AIChE J., 17, 335 (1971).

Vaporciyan, G. G., "Periodic Separating Reactors: Pressure Swing Adsorption with Reaction," Ph.D. Thesis, Univ. Michigan, Ann Arbor (1987).

Vaporciyan, G. G., and R. H. Kadlec, "Equilibrium-Limited Periodic Separating Reactors," AIChE J., 33(8), 1334 (1987).

Vaporciyan, G. G., A. Annapragada, and E. Gulari, "Rate Enhancements and Quasi-Periodic Dynamics During Forced Concentration Cycling of $\mathrm{CO}$ and $\mathrm{O}_{2}$ over Supported Pt-SnO ${ }_{2}$," Chem. Eng. Sci., 43, 2957 (1988).

Wankat, P. C., “Cyclic Separation Processes," Separ. Sci., 9(2), 85 (1974).

Manuscript received Feb. I and Apr. 8, 1988 in two parts, and revision received Jan. 20, 1989. 